\title{
HAADF STEM of Phase Separated Anion Exchange Membranes Prepared by Ultracryomicrotomy
}

\author{
Aaron C. Jackson ${ }^{1}$, Yifan $\mathrm{Li}^{2}$, Jacob R. Nyzaka ${ }^{3}$, Yossef A. Elabd ${ }^{3}$, Daniel M. Knauss ${ }^{2}$, Frederick L. \\ Beyer $^{1}$, Scott D. Walck ${ }^{4}$ \\ 1. U.S. Army Research Laboratory, Aberdeen Proving Ground, MD 21005 \\ 2. Colorado School of Mines, Golden, CO 80401 \\ 3. Drexel University, Philadelphia, PA 19104 \\ 4. Bowhead Technical Services, U.S. Army Research Laboratory, Aberdeen Proving Ground 21005
}

The study of the morphology of anion exchange membranes (AEMs) is important for developing more efficient alkaline fuel cells, sensors and purification systems. Both alkaline fuel cells and proton exchange membrane fuel cells (PEMFC) have high energy and power densities that make them great alternatives to the combustion engine in transportation applications. Alkaline fuel cells are cheaper than PEMFCs, but the mechanical and charge transport properties of AEMs are insufficient for widespread commercialization. To improve the properties of AEMs, new research is focused on optimizing the morphologies of the AEM.[1-3]

AEMs contain both hydrophobic and hydrophilic material in a morphology that is typically phase separated at the nanoscale. The hydrophilic regions contained bound ions that promote selective conductivity of counter ions, while the hydrophobic region provides mechanical stability for the membrane. Transmission electron microscopy (TEM) is an ideal analysis technique probing the morphology of these materials, particularly when combined with small-angle X-ray scattering.[1-3] In this work, the morphologies of different AEMs have been analyzed using high-angle annular dark field scanning TEM (HAADF STEM).

This work focused on a variety of AEMs based on block copolymers containing quaternary amines and one random copolymer containing metal-ligand complexes. In AEMs containing quaternary amines, the counter ion was bromine and maximized contrast in the TEM. The AEM containing metal-ligand complexes had inherently good contrast due to the presence of metal ions in its backbone. Perchlorate counter ions provided further contrast. Figure 1 shows the composition of these polymers.

The preparation of AEMs for analysis by TEM required precise control of many factors. From an environmental standpoint, humidity plays arguably the largest role in the morphology observed in the polymers. As a result, this work focuses on ensuring membranes are stained, sectioned, and stored under dry conditions. When sectioning $90 \mathrm{~nm}$ thick films, the temperature was a key variable. High temperatures resulted in soft films that stick to the cutting surface or deform during specimen transfer operations. At low temperatures, the AEMs used here had poor strength and were particularly susceptible to fracture at low temperature. As a result, a cryomicrotoming process was ideal for maintaining the ideal temperature, typically 10 degrees below the glass transition temperature $\left(T_{g}\right)$ of the membranes. Cryomicrotoming also offered the advantage of maintaining a dry atmosphere during the sectioning processes.

We used TEM, Energy Filtered TEM (EFTEM), X-ray energy dispersive spectroscopy (XEDS), and HAADF STEM to analyze the AEMs (Figure 2). TEM at $200 \mathrm{kV}$ with a small spot size and objective 
aperture provided adequate contrast for imaging. However, beam damage and insufficient elemental characterization required other TEM techniques to maximize the information obtained from the samples. Elemental mapping by EFTEM and XEDS mapping with HAADF STEM assigned elements to regions of the polymer and defects within each polymer. While beam damage also occurred using these techniques, low dose settings minimized that damage. Images taken using HAADF STEM had the best contrast with minimal beam damage. Based on all of the analytical techniques, we found that AEM-1 formed a lamellar morphology with large grain sizes, AEM-2 formed an ideal bi-continuous structure, and AEM-3 formed a new randomly distributed whisker morphology.

(a) AEM-1

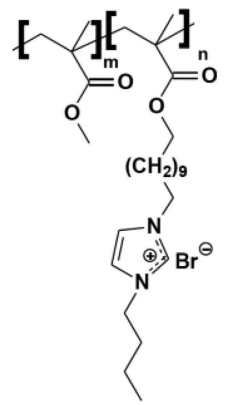

(c) AEM-3

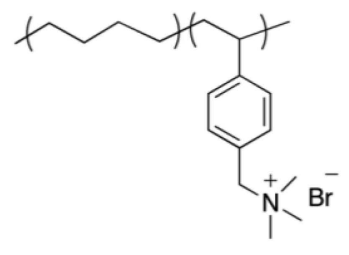

(b) AEM-2

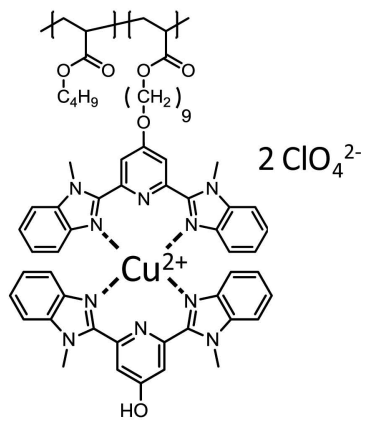

Figure 1. Structure of three AEM polymers. AEM-1 (a) is a block copolymer containing a poly(methyl methacrylate) block and an imidazolium based block. AEM-2 (b) is a block copolymer containing a poly(ethylene) block and a poly(vinyl benzyltrimethyl ammonium) block. AEM-3 is a random copolymer based on poly(butyl acrylate) containing grafted metal-ligand complexes.
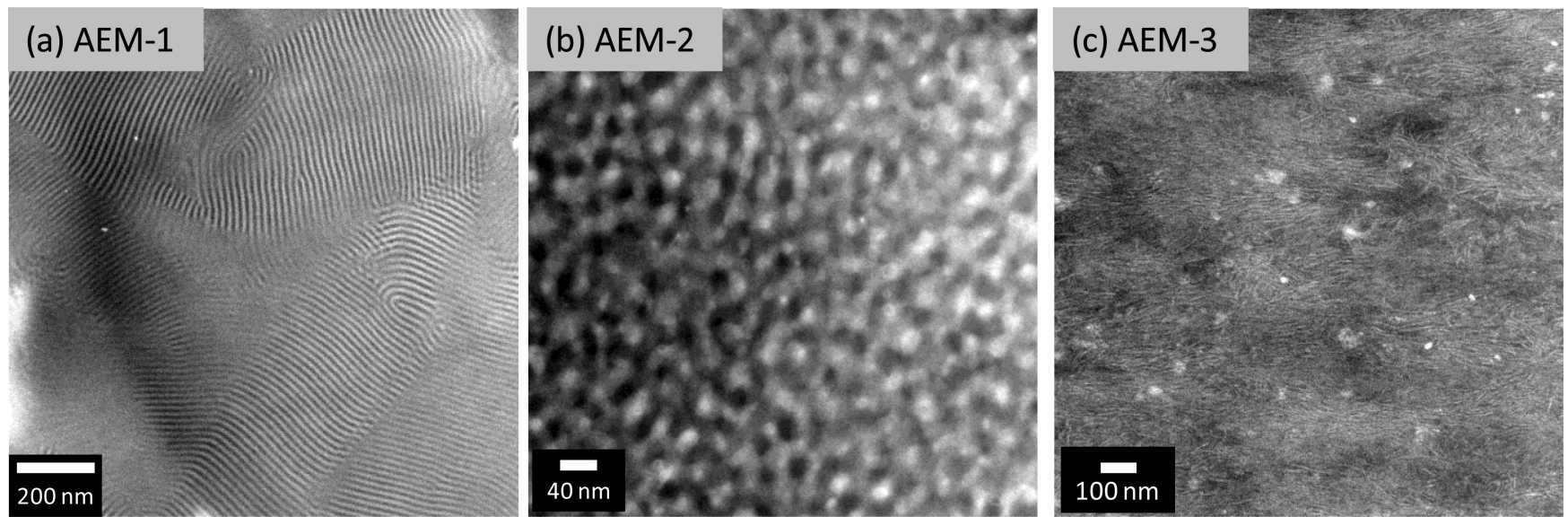

Figure 2. Representative HAADF STEM images of the AEMs studied.

[1] SC Price, et al., Macromolecules 46 (2013), p.7332-7340.

[2] AC Jackson, et al., Macromolecules 46 (2013), p. 5416-5422.

[3] Y Ye, et al., Macro Letters 2 (2013), p. 575-580.

[4] ACJ was supported through the Oak Ridge Institute of Science and Education through an interagency agreement between the U.S. Department of Energy and U.S. Army Research Laboratory (contract no. ORISE 1120-1120-99). 